BULLETIN Bulletin hispanique

HISPANIQUE Université Michel de Montaigne Bordeaux

118-1 | 2016

La Guerre Civile espagnole aujourd'hui (1936-2016)

\title{
Miguel de Cervantes, Comedias y tragedias
}

Real Academia Española, Madrid, 2015

Jean Canavaggio

\section{CpenEdition}

Journals

Édition électronique

URL : http://journals.openedition.org/bulletinhispanique/4440

DOI : 10.4000/bulletinhispanique.4440

ISSN : 1775-3821

Éditeur

Presses universitaires de Bordeaux

Édition imprimée

Date de publication : 15 juillet 2016

Pagination : 372-375

ISBN : 979-10-300-0058-0

ISSN : 0007-4640

Référence électronique

Jean Canavaggio, « Miguel de Cervantes, Comedias y tragedias », Bulletin hispanique [En ligne], 118-1 | 2016, mis en ligne le 15 juillet 2016, consulté le 23 septembre 2020. URL : http://

journals.openedition.org/bulletinhispanique/4440 ; DOI : https://doi.org/10.4000/bulletinhispanique. 4440

Ce document a été généré automatiquement le 23 septembre 2020

Tous droits réservés 


\section{Miguel de Cervantes, Comedias y tragedias}

Real Academia Española, Madrid, 2015

Jean Canavaggio

\section{RÉFÉRENCE}

Miguel de Cervantes, Comedias y tragedias, al cuidado de Luis Gómez Canseco, edición, estudio y anejos de Fausta Antonucci, Alfredo Baras Escolá, Sergio Fernández López, Ignacio García Aguilar, Luis Gómez Canseco, Valentín Núñez Reinoso, María del Valle Ojeda Calvo, Marco Presotto, José Manuel Rico García, Adrián Sáez, Debora Vaccari, Beatriz Pinzan y Martina Colombo. Madrid, Real Academia Española, 2015.

1 Saluons sans plus attendre la publication de cet ouvrage. Les deux tomes dont il se compose et qui viennent s'ajouter à celui des Entremeses, paru il y a quatre ans dans la même collection ${ }^{1}$, mettent enfin à notre disposition la meilleure édition à ce jour du théâtre de Cervantès. À ce titre, ils constituent une contribution fondamentale à notre connaissance et à notre compréhension de ce théâtre.

2 Tout en reconnaissant les mérites des précédents éditeurs - en particulier SchevillBonilla et, plus près de nous, Florencio Sevilla et Antonio Rey Hazas - et sans mésestimer sa dette envers une somme considérable de travaux dont la bibliographie finale réunit les titres sur près de 250 pages $^{2}$, Luis Gómez Canseco, avec le concours de ses douze collaborateurs, n'en a pas moins édifié un ensemble dont la richesse et la qualité s'imposent au lecteur, sans que le caractère collectif de l'entreprise qu'il a coordonnée en compromette le moins du monde la cohérence. Le tome $\mathrm{I}$, après une brève présentation liminaire, nous donne le texte annoté des Ocho comedias suivi de celui des trois pièces qui nous sont parvenues manuscrites : El trato de Argel, la Tragedia de Numancia et La conquista de Jerusalén por Godofre de Bullón, dont la probable paternité cervantine se voit ainsi admise. Le tome II, conformément aux normes de la collection, s'ouvre sur une étude préliminaire, signée de Luis Gómez Canseco et María del Valle 
Ojeda Calvo et intitulée «Cervantes y el teatro» (p.9-60). Une succession de «lectures» (p. 61-194) assure la présentation de chacune des pièces, comportant, le plus souvent, un découpage entre les différents aspects abordés. Viennent ensuite une « histoire du texte » (p. 195-217) qui distingue tour à tour tradition imprimée, tradition manuscrite et éditions modernes ; un exposé des critères retenus par Gómez Canseco et son équipe pour leur propre travail d'éditeurs (p. 218-222); une version condensée de l'apparat critique $^{3}$ (p. 223-283), une série de notes complémentaires (p. 285-653) qui, comme leur nom l'indique, complètent les notes de bas de page du tome I ; enfin quatre importantes annexes: résumé des différentes intrigues (p.657-675), tableau de la versification (p.677-694), relevé des représentations connues, des origines à 2013 (p. 695-709), fragments conservés des rôles d'acteurs dans le cas de El trato de Argel et de la Jerusalén (p. 711-765). La bibliographie (p. 767-903), l'index des notes (p. 905-943) et la table des matières parachèvent ce second volume.

On appréciera mieux l'intérêt de cette édition en évoquant quelques-unes des questions sur lesquelles son apport s'avère particulièrement utile. Tout d'abord l'établissement $\mathrm{du}$ texte des différentes pièces qui, dans le cas des Ocho comedias, s'appuie sur un examen rigoureux des exemplaires conservés de l'édition princeps, tandis que, dans celui des Obras sueltas, les éditeurs ont procédé à une comparaison serrée des différentes traditions manuscrites, à partir notamment des deux copies de la BNM avec le códice Sancho Rayón. Ensuite, l'hypothèse défendue naguère par Cotarelo Valledor, selon laquelle La casa de los celos, La gran sultana et El laberinto de amor seraient les refontes respectives de trois des pièces mentionnées en 1614 dans la "Adjunta al Parnaso " (El bosque amoroso, La confusa y La gran turquesca) : une hypothèse dont les éditeurs font justice au moins pour les deux premières (p. 74 y 125-126), tandis qu'en ce qui concerne La gran turquesca, Gómez Canseco n'exclut pas totalement cette possibilité (p.112). Puis, le problème épineux de la chronologie, que l'auteur de ce compte rendu avait tenté d'établir, il y a près de quarante ans, sur des bases plus ou moins conjecturales et qui bénéficie désormais d'un réexamen minutieux. On retiendra notamment, de ce point de vue, les dates proposées avec la prudence qui s'impose par Alfredo Baras Escolá pour Los baños de Argel et la Numancia, par Valentín Núñez Rivera pour El rufián dichoso, et par Luis Gómez Canseco pour La gran sultana. Enfin, le croisement entre histoire et fiction, dont le théâtre de Cervantès décline les différentes modalités à travers des variations qui ne se ramènent jamais à un schéma préétabli. Quant à la présentation que Fausta Antonucci nous donne de La conquista de Jerusalén, elle tient compte assurément des travaux antérieurs sur lesquels elle s'appuie (en particulier ceux de Stefano Arata et d'Héctor Brioso Santos); en même temps, elle enchaîne une série d'analyses qui, tout faisant apparaître la parenté de cette pièce avec l'ensemble du corpus dramatique cervantin, n'en soulignent pas moins son caractère spécifique, y compris dans ses imperfections mêmes.

On ne manquera pas d'être frappé, comme je l'ai été moi-même, par le titre - Comedias y tragedias - donné par les éditeurs à ces deux volumes. S'il fait écho à celui que Juan de la Cueva, en 1588, avait retenu pour l'édition de ses propres pièces, on peut se demander s'il convient parfaitement. Rappelons en effet que Cervantès, chaque fois qu'il se réfère à son théâtre, parle exclusivement des comedias dont il est l'auteur, y compris lorsqu'il cite la Numancia. Certes, il s'agit d'un choix rétrospectif, puisque cette étiquette apparaît dans des textes qu'il publie entre 1605 et 1615, alors que la Comedia nueva est en plein essor. Par ailleurs, alors que le manuscrit de la Numancia conservé à la Biblioteca Nacional de Madrid est appelé comedia, celui de la Hispanic Society, 
contemporain du précédent et qui ne sera édité qu'en 1784 par Antonio de Sancha, est intitulé tragedia. Toutefois, ce n'est qu'à partir du $\mathrm{xx}^{\mathrm{e}}$ siècle que cette pièce est unanimement qualifiée comme telle, et c'est alors que s'instaure un débat sur le statut précis auquel elle est censée correspondre: tragedia, tragicomedia ou tragedia de lieto fine $^{4}$. Par conséquent, à supposer même que ce débat soit désormais tenu pour clos, Gómez Canseco et ses collaborateurs auraient dû, en toute rigueur, intituler leur édition Comedias y tragedia. On comprend qu'ils se soient refusés à le faire. Il semble donc qu'ils aient choisi de tourner la difficulté en mettant l'accent, au fil de leurs analyses, sur la part d'éléments tragiques ou pathétiques que comportent certaines des autres pièces qui figurent dans ce volume. Ainsi, dans El trato de Argel, la présence sur scène de l'horreur en fonction d'un modèle théâtral qui s'apparente à celui de la tragedia patética (p. 166-169); dans Los baños de Argel, les morts violentes et les martyres représentés sur scène, qui restituent à l'action tout le sérieux dont Lope de Vega avait fait l'économie dans Los cautivos de Argel (p. 92-93); dans La gran sultana, les destins respectifs des captifs du sérail, pris dans une constante oscillation entre tragique et comique (p.117-120); dans El laberinto de amor, le triste sort de Rosamira qui, victime d'une fausse accusation, ne sait jusqu'au dénouement si elle échappera au châtiment qui pèse sur elle (p. 129-131). Toutefois, dès lors que Cervantès, dans cette optique, nous apparaît comme l'un de ceux qui forgent un nouveau modèle théâtral, irréductible à la division néo-aristotélicienne entre tragédie et comédie (p. 167), le titre retenu par les éditeurs se justifie encore moins. Peut-être eût-il été alors préférable d'adopter pour l'ensemble des trois volumes un titre neutre - «Cervantes, Teatro»- en spécifiant le contenu de chacun d'entre eux par l'énoncé des titres des pièces y figurant.

Le théâtre de Cervantès, on le sait, n'a subi qu'épisodiquement l'épreuve des planches, si bien qu'une ligne de partage sépare les œuvres qui, à différentes époques, ont eu la faveur des comédiens (La Numancia, Los baños de Argel, La gran sultana ou Pedro de Urdemalas) de celles qui, comme La casa de los celos ou El laberinto de amor, n'ont toujours pas retenu leur attention. De ce point de vue, les pages que Luis Gómez Canseco et María del Valle Ojeda, dans leur étude liminaire, consacrent à ses potentialités dramatiques et scéniques s'articulent heureusement avec le relevé que Martina Colombo et Beatriz Pinzan nous donnent des représentations connues, ainsi qu'avec les commentaires qu'appellent, de la part de Debora Vaccari, les fragments conservés des rôles d'acteurs. Quant au verdict porté par la postérité sur ce théâtre, les éditeurs, plutôt que de se borner à le ratifier ou à le contester, ont préféré à juste titre, le mettre en perspective. D'un côté, ils prennent acte de maladresses indiscutables qui tiennent à la fois aux tâtonnements du débutant qu'a été Cervantès au retour de sa captivité, à ses réticences à l'égard de la formule de la Comedia nueva dont il conteste la tendance à réduire la complexité du temps vécu à un schéma simple et efficace, enfin à son divorce avec le monde de la scène, dont il s'est éloigné en posant la plume pendant plusieurs années et avec lequel il n'a jamais pu renouer ses relations d'antan. De l'autre, ils considèrent que ces pièces nous offrent tout un éventail de possibilités expérimentales, propres à mettre en valeur la trajectoire de personnages qui, à la différence de ceux de Lope, s'inventent à mesure qu'ils se cherchent et se construisent à mesure qu'ils se découvrent. Ces possibilités, mises en lumière par le renouveau des études dont le théâtre de Cervantès fait l'objet depuis une quarantaine d'années, s'affirment désormais à la faveur du regain d'intérêt dont il commence à bénéficier de la part de comédiens désireux de le soumettre au banc d'essai de nouvelles dramaturgies. Il y a lieu de penser que les spécialistes du Siècle d'or réserveront le meilleur accueil à cette 
édition exemplaire, mais il faut aussi espérer que, dans tous les pays de langue espagnole, les praticiens de la scène sauront saisir l'occasion qu'elle leur offre.

\section{NOTES}

1. Miguel de Cervantes, Entremeses, edición, estudio y notas de Alfredo Baras Escolá, Madrid, Real Academia Española, 2012.

2. Parmi ces titres, ceux que j'ai publiés pour ma part depuis 1966 ont droit à une appréciation à laquelle je suis bien entendu vivement sensible.

3. L'apparat critique complet peut être consulté sur le web www.bcrae.es

4. On nous permettra, à ce sujet, de renvoyer le lecteur à notre récent article: «La Numancia, de comedia a tragedia ", Deste artife. Estudios en honor de Aldo Ruffinatto, ed. G. Carrascón y D. Capra, Alessandria, Edizioni dell'Orso, 2014, p. 83-91.

\section{AUTEURS}

JEAN CANAVAGGIO

Université de Paris X-Nanterre 